\title{
Nota
}

\section{HIDRÓXIDOS DUPLOS LAMELARES COMO MATRIZES PARA FERTILIZANTES DE LIBERAÇÃO LENTA DE NITRATO ${ }^{(1)}$}

\author{
Valentim da Silva ${ }^{(2)}$, Marcos Y. Kamogawa ${ }^{(3)}$, Rafael Marangoni ${ }^{(4)}$, Antonio Salvio \\ Mangrich $^{(5)}$ \& Fernando Wypych ${ }^{(2)}$
}

\begin{abstract}
RESUMO
Diante do extensivo uso de fertilizantes nitrogenados na agricultura e elevado potencial de lixiviação do nitrato, há uma demanda por fertilizantes de liberação lenta ou controlada. Os hidróxidos duplos lamelares (HDLs) sintéticos reúnem características que os qualifica para o uso como matrizes para fertilizantes de liberação lenta de íons nitrato. Os objetivos deste trabalho foram focados na síntese, caracterização e investigação do processo de liberação lenta de nitrato em um HDL contendo íons $\mathrm{Mg}^{2+}$ e $\mathrm{Al}^{3+}$ em sua estrutura. O HDL com a fórmula $\mathrm{Mg}_{0,83} \mathrm{Al}_{0,17}(\mathrm{OH})_{2}\left(\mathrm{NO}_{3}\right)_{0,17} \cdot 0,56 \mathrm{H}_{2} \mathrm{O}$ foi sintetizado pelo método de coprecipitação em pH alcalino constante, separado por centrifugação e seco em estufa a vácuo. As análises de difração de raios-X, de espectroscopia vibracional na região do infravermelho com transformada Fourier e as análises térmicas comprovam a obtenção do composto contendo o íon nitrato hidratado intercalado, além da fórmula proposta. $\mathrm{O}$ composto foi submetido a testes de liberação de nitrato em solução de $\mathrm{NaHCO}_{3}$ tamponado a pH 6,5 e em água destilada. As curvas obtidas evidenciaram dois eventos complementares de liberação de nitrato, um evento inicial rápido e um outro lento e gradativo. $O$ composto investigado neste trabalho demonstrou potencial para ser utilizado como matriz para fertilizante de liberação lenta de nitrato.
\end{abstract}

Termos de indexação: compostos de intercalação, reações de troca aniônica, barreira iônica interlamelar.

\footnotetext{
(1) Trabalho submetido para publicação em $1^{\circ}$ de junho de 2012 e aprovado em 25 de setembro de 2013.

(2) Laboratório de Química de Materiais Avançados - LAQMA, Universidade Federal do Paraná - UFPR. Caixa Postal 19081. CEP 81531-990 Curitiba (PR), Brasil. E-mail: valentimdasilva@gmail.com,wypych@ufpr.br

(3) Departamento de Ciências Exatas, Escola Superior de Agricultura "Luiz de Queiroz", Universidade de São Paulo. Caixa Postal 9. Av. Pádua Dias, 11. CEP 13418-900 Piracicaba (SP), Brasil. E-mail: kamogawa@usp.br

(4) Laboratório de Materiais e Compostos inorgânicos - LABMAT, Departamento de Química, Universidade Estadual do CentroOeste, Campus Cedeteg. Rua Simeão Camargo Varela de Sá, 3. CEP 85040-080 Guarapuava (PR), Brasil. E-mail: rmarangoni@unicentro.br

(5) Laboratório de Projetos e Processos Ambientais - LABPAM, Departamento de Química, UFPR. E-mail: mangrich@ufpr.br
} 


\title{
SUMMARY: LAYERED DOUBLE HYDROXIDES AS MATRIZES FOR NITRATE SLOW RELEASE FERTILIZERS
}

\begin{abstract}
Due to the extensive use of nitrogen fertilizers in the agriculture e due to the elevate potential of nitrate leaching, there is a great demand for slow or controlled release fertilizers. Synthetic layered double hydroxides (HDLs) combine the characteristic that qualifies them to be used as matrixes for nitrate slow release fertilizers. The objectives of the present work are focused in the synthesis, characterization and investigation of the slow release of nitrate process in one structure of a HDL containing ions $\mathrm{Mg}^{2+}$ and $\mathrm{Al}^{3+}$ in its structure. The HDL with the formula $\mathrm{Mg}_{0,83} \mathrm{Al}_{0,17}(\mathrm{OH})_{2}\left(\mathrm{NO}_{3}\right)_{0,17} \cdot 0,56 \mathrm{H}_{2} \mathrm{O}$ was synthesized by the co-precipitation method at constant alkaline $\mathrm{pH}$, isolated by centrifugation and dried in an vacuum oven. Measurements of $X$-ray diffraction (XRD), Fourier Transform Infrared spectroscopy (FTIR) and thermal analysis (TGA/DTA) confirmed the obtaining of the compound containing the intercalated hydrated nitrate anions and the proposed formula. The compound was tested for release nitrate $\mathrm{NaHCO}_{3}$ solution buffered to $\mathrm{pH}$ 6.5, and distilled water. The representative curves demonstrated two complementary behaviors nitrate release a fast initial and a slow gradual. The compound investigated in this study demonstrates the potential to be used as a matrix for slow release fertilizer nitrate. The compound was submitted to the nitrate release tests using a $\mathrm{NaHCO}_{3}$ solution buffered at pH 6.5 and in distilled water. The obtained curves have shown two complementary events of nitrate release, one fast initial event and another slow and gradual. The compound investigated in the present work has shown a potential to be used as matrix for nitrate slow release fertilizers.
\end{abstract}

Index terms: intercalation compounds, anionic exchange reactions, interlayer ionic barrier.

\section{INTRODUÇÃO}

O termo hidróxido duplo lamelar (HDL) é usado para designar compostos lamelares naturais ou sintéticos, que têm como estrutura básica a brucita $\left[\mathrm{Mg}(\mathrm{OH})_{2}\right]$. Esses compostos apresentam os cátions de $\mathrm{Mg}^{2+}$ coordenados octaedricamente por íons hidroxila. Os íons de $\mathrm{Mg}^{2+}$ podem ser substituídos isomorficamente por $\mathrm{Al}^{3+}$, gerando compostos com uma fórmula genérica do tipo $\left[\mathrm{M}^{2+}{ }_{1-\mathrm{x}} \mathrm{M}^{3+}{ }_{\mathrm{x}}(\mathrm{OH})_{2}\right]^{\mathrm{x}-}\left(\mathrm{A}^{-\mathrm{n}}\right)_{\mathrm{x} /}$ n. $\mathrm{yH}_{2} \mathrm{O}$, em que $\mathrm{M}^{2+}$ e $\mathrm{M}^{3+}$ representam, respectivamente, $\mathrm{Mg}^{2+} \mathrm{e} \mathrm{Al}^{3+}$, os cátions divalentes e trivalentes da lamela, e $\mathrm{A}^{-\mathrm{n}} \cdot \mathrm{yH}_{2} \mathrm{O}$ representa um ânion hidratado intercalado (Olanrewaju et al., 2000).

Os HDLs podem ser sintetizados com ampla diversidade de composições químicas, gerando compostos que diferem com a variedade de íons metálicos divalentes e trivalentes, com as relações molares $\mathrm{M}^{2+} / \mathrm{M}^{3+}$ e com a diversidade de ânions intercalados. Ainda, são várias as técnicas de síntese empregadas, sendo mais comuns aquelas que utilizam a coprecipitação em $\mathrm{pH}$ alcalino constante (Arizaga et al., 2007; Olfs et al., 2009; Marangoni et al., 2009). Os métodos de síntese, as propriedades, as possíveis composições, as modificações químicas de suas superfícies, bem como as aplicações dessa classe de materiais, são apontadas na literatura (Bull; 2001; Komarneni et al., 2003; Arizaga et al., 2007; Marangoni et al., 2009; Torres-Dorante et al., 2009).

Em razão da capacidade de troca aniônica dos HDLs, que usualmente varia entre 200 e 450 $\mathrm{cmol}_{\mathrm{c}} \mathrm{kg}^{-1}$ (Bull, 2001), e do baixo custo de síntese, busca-se ampliar o potencial de formulações dos HDLs para que possam ser utilizados como fertilizantes de liberação controlada ou lenta, especialmente de nitrogênio $\left(\mathrm{NO}_{3}{ }^{-}\right)$, fósforo $\left(\mathrm{H}_{2} \mathrm{PO}_{4}{ }^{-}\right.$ou $\left.\mathrm{HPO}_{4}{ }^{2-}\right)$ e enxofre $\left(\mathrm{SO}_{4}{ }^{2-}\right)$. Os HDLs são perfeitamente factíveis para os propósitos agronômicos, considerando a sua ocorrência na forma de minerais e baixa solubilidade, embora os elementos $\mathrm{M}^{2+} \mathrm{e}^{3+}$, que compõem as suas estruturas, possam ser considerados nutrientes das plantas, além de serem de forma geral atóxicos, não influenciando o meio ambiente.

No que se refere à liberação de $\mathrm{N}$, especificamente do $\mathrm{NO}_{3}{ }^{-}$, estudos que desenvolvem e aprimoram os mecanismos de controle da sua retenção e liberação contribuem para minimizar o impacto desse ânion em águas superficiais e subterrâneas e para melhorar o sincronismo entre a disponibilidade de $\mathrm{N}$ e a demanda das plantas. Ressalta-se que o limite de tolerância de $\mathrm{NO}_{3}{ }^{-}$na água para consumo animal é de $10 \mathrm{mg} \mathrm{L}^{-1} \mathrm{de}$ acordo com a legislação brasileira e de $6 \mathrm{mg} \mathrm{L}^{-1}$, para a legislação paulista (Alaburda \& Nishihara, 1998). Além de contaminar o meio ambiente, a lixiviação do $\mathrm{NO}_{3}$ ' é de grande relevância econômica, pois contribui para a perda do $\mathrm{N}$ aplicado via fertilizante, que pode atingir de 40 a $70 \%$ do total aplicado, como ressaltado por Wu \& Liu (2008) com a utilização de fertilizantes nitrogenados convencionais nos sistemas agrícolas.

Alguns trabalhos têm descrito o potencial dos HDLs como agentes de liberação lenta (Olanrewaju et al., 2000; Komarneni et al., 2003; Torres-Dorante et al., 2009). Dessa forma, a utilização dos compostos lamelares como matrizes para obtenção de fertilizantes de liberação lenta de $\mathrm{NO}_{3}{ }^{-}$pode oferecer alternativas no que se referem aos aspectos agronômicos, relacionado à redução do consumo de fertilizantes nitrogenados convencionais e, ainda, minimizar a 
carga poluente nas águas e no ambiente, possibilitando desse modo benefícios agrícolas e ambientais (Wu \& Liu, 2008).

Considerando-se os diversos mecanismos de natureza biológica ou química que disponibilizam o $\mathrm{NO}_{3}{ }^{-}$na solução do solo, os HDLs podem também contribuir para elevar o poder tampão desse ânion no solo.

Os objetivos deste trabalho foram produzir e caracterizar um HDL intercalado com o íon nitrato

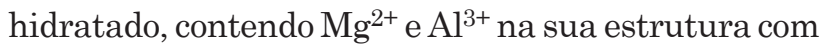
razão molar próxima a $5\left(\mathrm{Mg}^{2+}: \mathrm{Al}^{3+}\right)$ e investigar o comportamento da sua liberação em uma solução aquosa de íons $\mathrm{HCO}_{3}{ }^{-}$e em água destilada.

\section{MATERIAL E MÉTODOS}

\section{Síntese do HDL-Mg $\mathrm{Mg}_{5} \mathrm{Al}_{1}$ com $\mathrm{NO}_{3}$ - intercalado}

O HDL foi obtido pelo método de coprecipitação a $\mathrm{pH}$ alcalino constante (Olfs et al., 2009); todo o processo de síntese foi conduzido em atmosfera de $\mathrm{N}_{2}$. Utilizaram-se $21,49 \mathrm{~g}$ de $\mathrm{Mg}\left(\mathrm{NO}_{3}\right)_{2} \cdot 6 \mathrm{H}_{2} \mathrm{O}$ e $6,44 \mathrm{~g}$ de $\mathrm{Al}\left(\mathrm{NO}_{3}\right)_{3} .9 \mathrm{H}_{2} \mathrm{O}$ para obtenção da relação molar de $5: 1$ de $\mathrm{Mg}^{2+} / \mathrm{Al}^{3+}$. Os sais foram dissolvidos em $50 \mathrm{~mL}$ de água destilada e desaerada, para evitar contaminação com íons $\mathrm{CO}_{3}{ }^{-2}$ provenientes do $\mathrm{CO}_{2}$ atmosférico dissolvido na água. $\mathrm{O}$ volume foi completado para $125 \mathrm{~mL}$ e essa solução foi gotejada lentamente em uma solução de $\mathrm{NaNO}_{3}$, que continha quantidade de $\mathrm{NO}_{3}{ }^{-}$suficiente para que a relação molar com $\mathrm{M}^{3+}$ fosse quatro vezes maior do que a necessária para a intercalação do íons $\mathrm{NO}_{3}$, o qual é proporcional ao teor de íons $\mathrm{Al}^{3+}$ na estrutura. Simultaneamente à adição de sais, gotejou-se, sobre a solução de $\mathrm{NaNO}_{3}$, uma solução de $\mathrm{NaOH} 0,2 \mathrm{~mol} \mathrm{~L}^{-1}$ (Arizaga et al., 2007), sendo o $\mathrm{pH}$ fixado durante a síntese em próximo de 10, considerado adequado para obtenção de HDL$\mathrm{Mg}_{5} \mathrm{Al}_{1}$. O meio reacional em temperatura ambiente foi constantemente homogeneizado com agitador magnético; o composto formado foi retirado pelo frasco de resgate e lavado cinco vezes com água destilada para remover o excesso do $\mathrm{NO}_{3}{ }^{-}$. Em seguida, o sólido foi separado por centrifugação a $3.056 \mathrm{~g}$ por $12 \mathrm{~min}$ e seco em estufa a vácuo, a $60^{\circ} \mathrm{C}$ até peso constante, e após. Para desagregar o gel formado, o material foi submetido à moagem por $30 \mathrm{~min}$, em um moinho de esferas, obtendo-se o composto em pó de dimensões submicrométricas, para posteriores caracterizações.

\section{Caracterização}

As análises de difração de raios-X (XRD) foram realizadas em um difratômetro Shimadzu modelo XRD-6000, utilizando-se tubo de raios-X de $\mathrm{Cu}$ $(\lambda=0,15418 \mathrm{~nm} ; 30 \mathrm{~mA}$ e $40 \mathrm{kV})$; os dados foram coletados na faixa de $3,0^{\circ}$ a $70,0^{\circ}(2 \theta)$, com velocidade de varredura de $1^{\circ} \mathrm{min}^{-1}$ e passo de $0,02^{\circ}$. As amostras foram acondicionadas em porta-amostras de vidro neutro e levemente prensadas.

Os espectros de infravermelho por transformada de Fourier (FTIR) foram coletados em um espectrofotômetro Bomem Michelson MB100, utilizando pastilhas de $\mathrm{KBr}$, prensadas a $10 \mathrm{t}$, e contendo $1 \%$ de amostra. As análises foram realizadas no modo de transmissão na faixa de $4.000-400 \mathrm{~cm}^{-1}$, com resolução de $8 \mathrm{~cm}^{-1}$ e acumulação de 32 varreduras.

As medidas de análise térmica (TGA/DTA) foram realizadas em equipamento Mettler Toledo TGA/S-DTA $851 \mathrm{E}$, utilizando-se cadinhos de platina, sob fluxo $50 \mathrm{~mL} \mathrm{~min}^{-1}$ de oxigênio, à taxa de aquecimento de $10{ }^{\circ} \mathrm{C}$ min $^{-1}$.

As análises químicas para determinação do teor total de $\mathrm{NO}_{3}$ - foram realizadas a partir das amostras digeridas em meio ácido, utilizando-se $8 \mathrm{~mL}$ de $\mathrm{HCl}$ p.a.. Para estudar o mecanismo de liberação dos íons $\mathrm{NO}_{3}$, utilizou-se 0,25 g de HDL-MgAl, completando o volume a $50 \mathrm{~mL}$ de solução tamponada em $\mathrm{pH}$ 6,5 de $\mathrm{NaHCO}_{3}$ ou em água com $\mathrm{CO}_{3}{ }^{2-}$, decorrente do equilíbrio com o $\mathrm{CO}_{2}$ atmosférico. $\mathrm{O}$ teor de $\mathrm{NO}_{3}{ }^{-}$foi determinado por análise por injeção em fluxo (FIA), com o uso de um equipamento ASIA da Ismatec (Suíça) (Kamogawa \& Teixeira, 2009). As amostras foram analisadas no intervalo de tempo de 0,$5 ; 15 ; 30 ; 60$; $300 ; 1.440 ; 2.880 ; 4.320$; e 4.680 min para a solução de $\mathrm{HCO}_{3}$ - tamponado a $\mathrm{pH} 6,5$ e de 0,$5 ; 15 ; 30 ; 60$; $300 ; 1.440 ; 3.600 ; 5.400 ; 7.200$; e 9.000 min para a condição de liberação em água com $\mathrm{CO}_{3}{ }^{2-}$, decorrente do equilíbrio do ar atmosférico.

\section{Modelo matemático aplicado}

As análises de comportamento das curvas de liberação dos íons $\mathrm{NO}_{3}$ - para as duas soluções seguiram o modelo de Cabrera reparametrizado por Zeviani (2009). Com base nas análises descritivas e gráficas, adequou-se o modelo proposto com auxilio do software R (R Development Core Team, 2011), com a função nls, que implementa o algoritmo Gauss-Newton, utilizado para ajustar modelo não linear. A aplicação desse Modelo considera coeficientes associados às taxas de cada fase, com a finalidade de interpretação química do processo. A equação de ajuste utilizada foi $\left[\mathrm{NO}_{3}{ }^{-}\right]=\mathrm{A}\left(1-\mathrm{e}^{-\ln (2) . t / \mathrm{V}}\right)+\mathrm{D} . t$, em que $\left[\mathrm{NO}_{3}\right]_{l}=$ concentração de nitrato liberado no tempo $t ; A=$ taxa de nitrato liberado no momento rápido; $t=$ tempo de liberação; $V=$ velocidade de liberação de nitrato; e $D=$ taxa de nitrato liberado no momento lento.

\section{RESULTADOS E DISCUSSÃO}

Os picos obtidos do difratograma de raios-X do composto $\mathrm{HDL}-\mathrm{Mg}_{5} \mathrm{Al}_{1}$ (Figura 1a) foram indexados, considerando-se um sistema hexagonal com simetria 
romboédrica (grupo espacial R-3m). A partir do pico (110), foi possível determinar a distância interatômica metal-metal da lamela $(3,157 \AA)$, estando de acordo para a relação de $\mathrm{M}^{2+} / \mathrm{M}^{3+}$ proposta. O valor do espaçamento basal $(\mathrm{d}=8,22 \AA)$, obtido a partir do pico da maior ordem possível (009), subtraído do "d" da brucita ( $\mathrm{d}=4,78 \AA$ ), fornece a diferença de espaçamento basal $(\Delta \mathrm{d}=3,44 \AA)$, originado pela substituição do $\mathrm{Mg}$ pelo $\mathrm{Al}$ nas lamelas. Considerandose que o íon $\mathrm{NO}_{3}{ }^{-}$é um íon plano e na forma deitada é
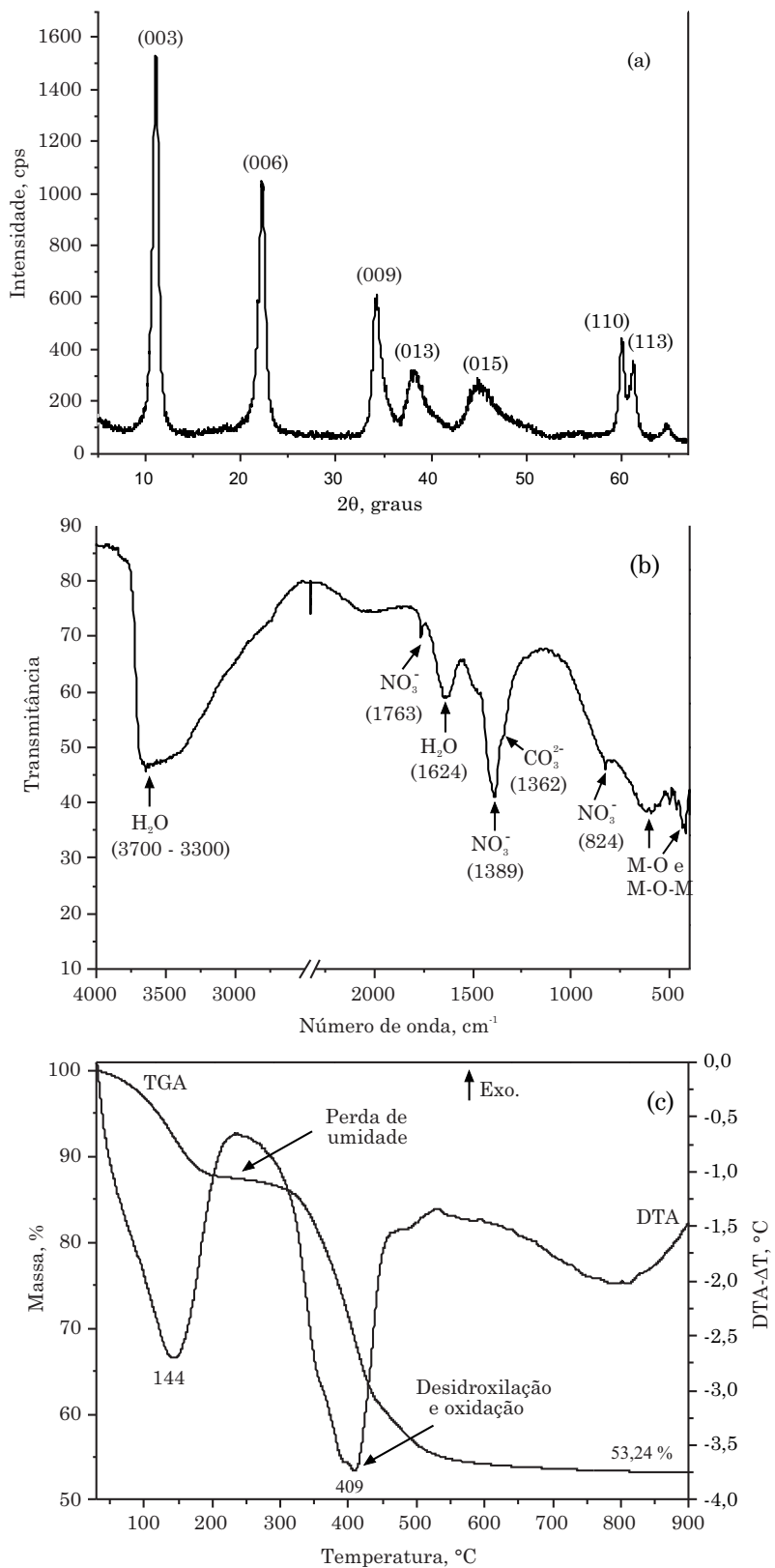

Figura 1. Caracterização do hidróxido duplo lamelar - HDL-Mg ${ }_{5} \mathrm{Al}_{1}$ : (a) difratograma de raios-X; (b) espectro vibracional na região do infravermelho com transformada de Fourier; e (c) curvas da análise térmica (análise termogravimétrica TGA e da análise térmica diferencial - DTA). equivalente a um átomo de oxigênio e ao longo do seu maior eixo (N-O) possui diâmetro de $4,80 \AA$ e a diferença obtida no espaçamento basal é inferior a esse valor, sugere-se que a intercalação do ânion esteja com o eixo ao longo do eixo N-O na orientação inclinada, em relação à lamela.

A presença e a forma dos picos (003), (006) e (009) indicam que há cristais pequenos com poucas lamelas empilhadas ao longo desse eixo cristalográfico (alguns angstrons); ainda, com os picos largos e assimétricos indexados com os índices (013) e (015) é permitido inferir que o empilhamento das lamelas ocorre de forma irregular, as quais se encontram rotacionadas ao longo do eixo basal.

A medida de espectroscopia vibracional na região do infravermelho com transformada de Fourier (FTIR) do composto HDL- $\mathrm{Mg}_{5} \mathrm{Al}_{1}$ (figura $1 \mathrm{~b}$ ) evidenciou os estiramentos simétricos $(v 3)$ de $\mathrm{N}-\mathrm{O}\left(\mathrm{NO}_{3}{ }^{-}\right)$na região de $1.389 \mathrm{~cm}^{-1}$, banda essa esperada para o íon $\mathrm{NO}_{3}$ livre (simetria $\mathrm{D}_{3 \mathrm{~h}}$ ), intercalado entre as lamelas, e em 824 e $1.763 \mathrm{~cm}^{-1}$ com simetria mais baixa $\left(\mathrm{C}_{2 v}\right)$. Embora a reação de precipitação tenha sido conduzida em atmosfera de $\mathrm{N}_{2}$, e utilizando-se água desaerada, vibrações de estiramento assimétrico do íon carbonato intercalado foram verificadas na banda em $1.362 \mathrm{~cm}^{-1}$, ocasionando um alargamento na região da banda de $\mathrm{NO}_{3}{ }^{-}$. As vibrações em $1.624 \mathrm{~cm}^{-1}$ são atribuídas à deformação angular das moléculas de água adsorvidas ou intercaladas. Na região de $3.400-3.700 \mathrm{~cm}^{-1}$, as bandas vibracionais são de grupamentos de hidroxilas e das moléculas de água, solvatando os íons $\mathrm{NO}_{3}{ }^{-}$. As absorções na região de $450-700 \mathrm{~cm}^{-1}$ são atribuídas às flexões M-O-M e aos estiramentos dos grupos M-O presente nas lamelas. As diferentes bandas vibracionais para o íon $\mathrm{NO}_{3}{ }^{-}$ainda podem estar relacionadas às diferentes coordenações possíveis $\left(3 \mathrm{Mg}^{2+}, 2 \mathrm{Mg}^{2+}+\mathrm{Al}^{3+}, \mathrm{Mg}^{2+}+2 \mathrm{Al}^{3+}\right.$ e $\left.3 \mathrm{Al}^{3+}\right)$, entre os arranjos dos octaedros nas lamelas da estruturas.

O comportamento térmico por DTA da estrutura de $\mathrm{HDL}-\mathrm{Mg}_{5} \mathrm{Al}_{1}$ (figura 1c) apresentou o primeiro pico endotérmico em $144^{\circ} \mathrm{C}$, referente aos eventos de perda de massa de água fisisorvidas e cointercaladas aos íons $\mathrm{NO}_{3}$. Esse primeiro evento, em temperatura acima de $100{ }^{\circ} \mathrm{C}$, caracterizou a existência de fortes ligações com a água e o aumento na estabilidade estrutural do HDL, pois necessitou de mais energia no sistema para que ocorram os eventos. $\mathrm{O}$ segundo pico endotérmico observado em $409{ }^{\circ} \mathrm{C}$ foi atribuído aos eventos de desidroxilação da matriz e liberação dos ânions intercalados, gerando 53,24 \% de óxidos de $\mathrm{MgO}$ e $\mathrm{Al}_{2} \mathrm{O}_{3}$. Em razão dos dados obtidos, estimou-se a fórmula $\mathrm{Mg}_{0,83} \mathrm{Al}_{0,17}(\mathrm{OH})_{2}\left(\mathrm{NO}_{3}\right)_{0,17} \cdot 0,56 \mathrm{H}_{2} \mathrm{O}$, com relação $\mathrm{Mg} / \mathrm{Al}$ de 4,88, próximo de 5,0, utilizado na estequiometria de síntese. $\mathrm{O}$ teor de $\mathrm{NO}_{3}$ - obtido a partir da fórmula proposta foi de $13,3 \%$, que é próximo do valor determinado por injeção de fluxo de $11,1 \%$. Essa pequena diferença pode ser atribuída à intercalação dos íons carbonato, que foram observados 
por FTIR e não foram considerados na fórmula do composto, além da variação do teor de umidade de amostra, que foi exposta ao meio ambiente em diferentes condições antes das análises.

\section{Liberação de nitrato em solução}

O HDL sintetizado foi submetido a processos de liberação de $\mathrm{NO}_{3}{ }^{-}$em solução de $\mathrm{NaHCO}_{3}$ tamponado a $\mathrm{pH} 6,5$ por $150 \mathrm{~h}$ e em água destilada, por $72 \mathrm{~h}$. Nessas condições, espera-se a troca no $\mathrm{NO}_{3}$ pelos íons de $\mathrm{HCO}_{3}$. Para a condição de liberação em água destilada, ressalta-se que com a adição dos HDLs ocorreu a elevação do $\mathrm{pH}$ para valores superiores a 9 , em razão da dissociação dos hidróxidos de metais das estruturas. Nessas condições de $\mathrm{pH}$, geram-se íons de $\mathrm{CO}_{3}{ }^{2-}$ que trocariam com os íons de $\mathrm{NO}_{3}{ }^{-}$.

As curvas que representam o comportamento de liberação do $\mathrm{NO}_{3}{ }^{-}$em solução estão representadas por dois momentos distintos e complementares: o primeiro, o momento de liberação rápida $(A)$; e, o segundo, o de liberação lenta $(D)$. A liberação inicial rápida está ligada ao $\mathrm{NO}_{3}$ - presente nas estruturas que é prontamente trocado em razão da alta concentração de ânion $\mathrm{HCO}_{3}{ }^{-}$ou $\mathrm{CO}_{3}{ }^{2-}$ e é seguida de uma liberação lenta, que se declina gradativamente até o equilíbrio no sistema.

A equação de ajuste aplicada $\left[\mathrm{NO}_{3}^{-}\right]_{l}=\mathrm{A}\left(1-\mathrm{e}^{-\ln (2) . t / \mathrm{V}}\right)$ + D.t (Zeviani, 2009) considera os dois segmentos, sendo possível apresentar numericamente os índices de liberação rápida e lenta de $\mathrm{NO}_{3}$. Observou-se na figura 2 a que a concentração total de $\mathrm{NO}_{3}{ }^{-}$, passível de liberação rápida, correspondeu a $30 \%$ do total presente na amostra. Com a taxa de liberação lenta de $0,49 \%$ no segundo momento, notou-se o comportamento gradativo de liberação de $\mathrm{NO}_{3}-\mathrm{O}$ mecanismo de troca lenta é atribuído a bloqueios periféricos nos espaços interlamelares das estruturas. A taxa de meia-vida (Tmv) de 44,49 h demonstra a resistência à troca aniônica das estruturas no meio. Ainda, conforme sugeridos por difração de raios-X, os diferentes arranjos implicaram na não homogeneidade da lamela, podendo favorecer, nessa situação, a liberação de $\mathrm{NO}_{3}$; os pontos em que apresentam $3 \mathrm{Al}^{3+}$, considerado ponto "neutro", facilitam a liberação do íon intercalado. Essas configurações, conciliando pequeno tamanho dos cristais, determinaram o teor de $\mathrm{NO}_{3}-$ liberado no momento rápido.

Verifica-se na figura $2 \mathrm{~b}$ que em água as trocas no momento rápido foram mais intensas (63\%). Esse comportamento está relacionado ao deslocamento causado pelos ânions de $\mathrm{CO}_{3}{ }^{2-}$, por esses possuírem dupla carga negativa, deslocando com maior facilidade os íons de $\mathrm{NO}_{3}$ - intercalado e por apresentarem maior afinidade pelas lamelas do HDL. O Tmv $=2,9 \mathrm{~h}$ demonstra que $50 \%$ do total dos íons $\mathrm{NO}_{3}$ - intercalados foram liberados rapidamente. Os intervalos de confiança (IC: 95 \%) apresentaram variação de mais ou menos $10 \%$ na fase de liberação lenta, em valores muito próximos da média da fase de liberação rápida. Esses resultados evidenciam que o ânion divalente libera maiores concentrações de $\mathrm{NO}_{3}$ - do que o ânion monovalente $\left(\mathrm{HCO}_{3}^{-}\right)$.

No momento lento, a taxa de liberação em média foi de $0,2 \%$, o que evidenciou que a relação estabelecida com o momento rápido é inversamente proporcional. Isso indica que os íons $\mathrm{NO}_{3}$. intercalados são, num momento inicial, trocados preferencialmente nas regiões periféricas dos cristais do HDL, dificultando as trocas dos íons de $\mathrm{NO}_{3}{ }^{-}$, que ainda se encontram nas regiões centrais do espaço interlamelar das estruturas. Esse comportamento de "bloqueio de íons" justifica a alta liberação de $\mathrm{NO}_{3}{ }^{-}$no momento rápido e o menor valor da taxa de liberação lenta.

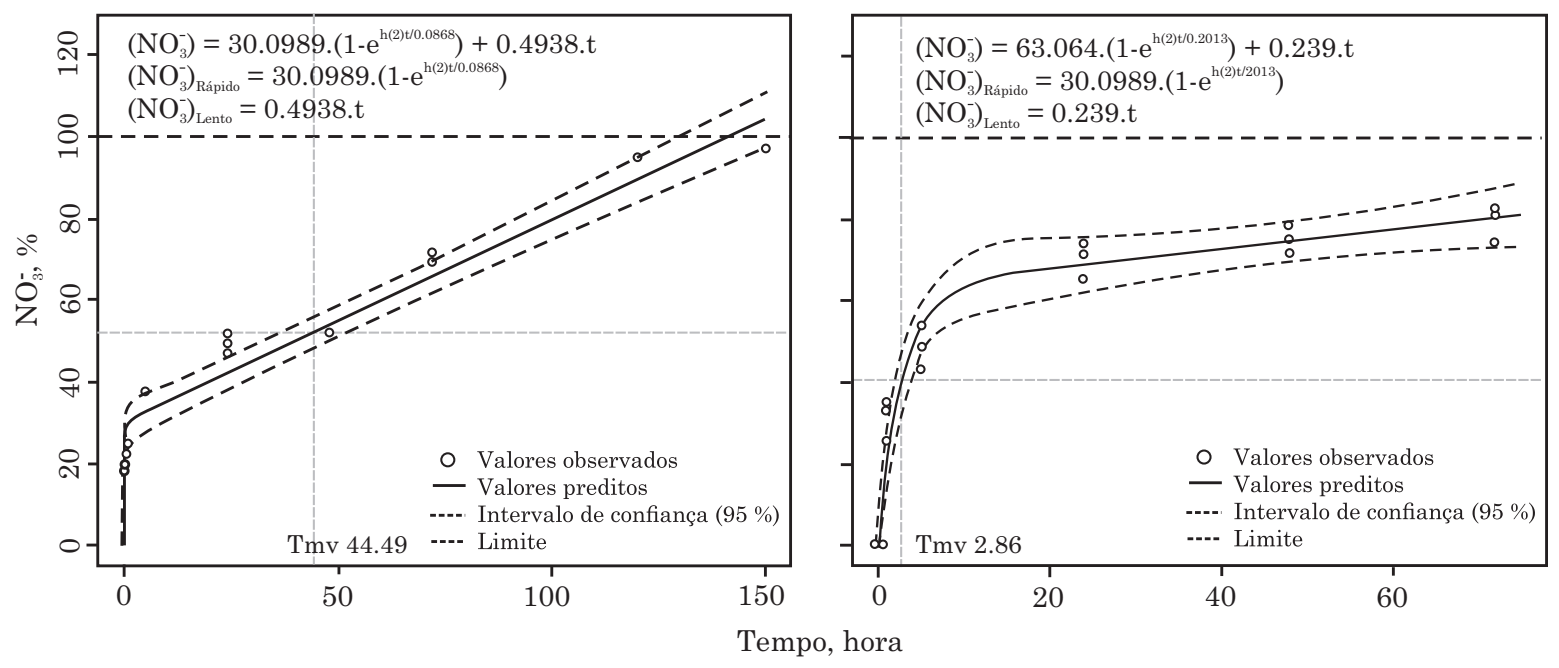

Figura 2. Curvas de liberação de nitrato em (a) solução de $\mathrm{NaHCO}_{3}$ tamponado a pH $6,5 \mathrm{em} 150 \mathrm{~h}$ e (b) solução em água em 72 h. 


\section{CONCLUSÕES}

1. O método de síntese de coprecipitação em $\mathrm{pH}$ alcalino constante é satisfatório para obtenção do HDL- $\mathrm{Mg}_{5} \mathrm{Al}_{1}$.

2. A valência dos ânions na solução influencia a liberação de nitrato para a solução; assim, o ânion carbonato (divalente) tem maior potencial de troca do que o ânion bicarbonato (monovalente).

3. Os resultados de liberação de nitrato em solução revelaram que HDL- $\mathrm{Mg}_{5} \mathrm{Al}_{1}$ é uma matriz promissora para obtenção de fertilizantes de liberação lenta de nitrato.

\section{AGRADECIMENTOS}

Aos diferentes suportes técnicos e apoio de profissionais, dentre os quais se destacam: Luis Omar Torres-Dorante, do Centro de Pesquisa de Hanninghof da YARA Internacional, Alemanha; Laboratório de Análises de Combustíveis Automotivos - LACAUT, em nome de Lílian Cristina Côcco e de Mitsuka Matsui; aos Laboratórios de Mineralogia e de Fertilidade do Departamento de Solos do Setor Agrárias da UFPR, em nome dos professores Vander de Freitas Melo e Antônio C. V. Motta; ao Departamento de Ciências Exatas - Química da Universidade de São Paulo (ESALQ), em nome do Prof. Marcos Kamogawa; e à UFPR Setor Litoral.

\section{LITERATURA CITADA}

ARIZAGA, G.G.C.; SATYANARAYANA, K.G. \& WYPYCH, F. Layered hydroxide salts: Synthesis, properties and potential applications. Solid State Ionics, 178:1143-1162, 2007.

ALABURDA, J. \& NISHIHARA, L. Presença de compostos de nitrogênio em águas de poços. R. Saúde Públ., 32:160$165,1998$.
BULL, C. Development and evaluation of Layered Double Hydroxides (LDHs) for nitrate exchange in soil. Germany, University of Hannover, 2001. (Tese de Doutorado)

KAMOGAWA, M.Y. \& TEIXEIRA, M.A. Autoamostrador de baixo custo para análise por injeção em fluxo. Quím. Nova, 32:1644-1646, 2009

KOMARNENI, S.; NEWALKAR, B.; LI, D.; GHEYI, T.; LOPANO, C.; HEANEY, P. \& POST, J. Anionic clays as pontential slow-release fertilizers: Nitrate ion exchange. J. Porous Mater., 10:243-248, 2003.

MARANGONI, R.; BOUHENT, M.; TAVIOT-GUEHO, C.; WYPYCH, F. \& LEROUXC, F. $\mathrm{Zn}_{2} \mathrm{Al}$ layered double hydroxides intercalated and adsorbed with anionic blue dyes: A physico-chemical characterization. J. Colloid Inter. Sci., 333:120-127, 2009.

OLANREWAJU, J.; NEWALKAR, B.L.; MANCINO, C. \& KOMARNENI, S. Simplified synthesis of nitrate form of layered double hydroxide. Mater. Lett., 45:307-310, 2000.

OLFS, H.W.; TORRES-DORANTE, L.O.; ECKELT, R. \& KOSSLICK, H. Comparison of different synthesis for $\mathrm{Mg}-\mathrm{Al}$ Layered Double Hydroxides (LDH): Characterization of the structural phases and anion exchange properties. Appl. Clay Sci., 43:459-464, 2009.

R. DEVELOPMENT CORE TEAM. R: A language and environment for statistical computing. R Foundation for Statistical Computing. Vienna, 2011. ISBN 3-900051-070, 2011. Disponível em: <http://www.R-project.org>. Acesso em: 24 Out. 2011.

TORRES-DORANTE, L.O.; LAMMEL, J. \& KUHLMANN, H. Use of a Layered Double Hydroxide (LDH) to buffer nitrate in soil: Long-term nitrate exchange properties under cropping and fallow conditions. Plant Soil, 315:257$272,2009$.

WU, L. \& LIU, M. Preparation and properties of chitosancoated NPK compound fertilizer with controlled-release and water-retention. Carbohydr. Poly., 72:240-247, 2008.

ZEVIANI, W.M. Avaliação de modelos de regressão não linear na cinética de liberação de potássio de resíduos orgânicos. Lavras, Universidade Federal de Lavras, 2009. 85p. (Dissertação de Mestrado) 